\title{
A Mechanochemical Model for the Simulation of Molecules and Molecular Crystals Under Hydrostatic Pressure
}

\author{
Tim Stauch*,1,2,3 \\ ${ }^{1}$ University of Bremen, Institute for Physical and Theoretical Chemistry, Leobener Straße NW2, D-28359 Bremen, Germany \\ ${ }^{2}$ University of Bremen, Bremen Center for Computational Materials Science, Am Fallturm 1, D-28359 Bremen, Germany \\ ${ }^{3}$ University of Bremen, MAPEX Center for Materials and Processes, Bibliothekstraße 1, D-28359 Bremen, Germany
}

\begin{abstract}
A novel mechanochemical method for the simulation of molecules and molecular crystals under hydrostatic pressure, the eXtended Hydrostatic Compression Force Field (X-HCFF) approach, is introduced. In contrast to comparable methods, the desired pressure can be adjusted non-iteratively and molecules of general shape retain chemically reasonable geometries even at high pressures. The implementation of the X-HCFF approach is straightforward and the computational cost is practically the same as for a regular geometry optimization. Pressure can be applied by using any desired electronic structure method for which a nuclear gradient is available. The results of X-HCFF for pressure-dependent intramolecular structural changes in the investigated molecules and molecular crystals as well as a simple pressure-induced dimerization reaction are chemically intuitive and fall within the range of other established computational methods. Experimental spectroscopic data of a molecular crystal under pressure are reproduced accurately.
\end{abstract}

\footnotetext{
* Corresponding author. Email: tstauch@uni-bremen.de
} 


\section{Introduction}

The field of high-pressure chemistry has seen tremendous progress within the past few decades. ${ }^{1}$ Pressureinduced changes in the rate, mechanism and equilibrium of chemical reactions have opened up new avenues in chemical synthesis. ${ }^{2,3}$ Using diamond anvil cells, ${ }^{4}$ pressures of several hundred GPa have been realized experimentally, meaning that pressures as high as in Earth's core are now available in the laboratory. ${ }^{5}$ Not surprisingly, the geometries of molecules, ${ }^{6-8}$ the lattice constants of crystals ${ }^{9-11}$ and crystal phases ${ }^{12}$ have been found to change under pressure. However, many of the pressure-induced structural changes of molecules and crystals can only be inferred indirectly by interpreting the concomitant changes in the spectroscopic properties, e.g., the wavelengths and intensities of absorption.

As a result, the demand for computational tools that predict structural and spectroscopic properties of molecules as well as chemical reactivity at high pressure is increasing. Methods that model the influence of pressure on periodic chemical systems have been described in the realm of Molecular Dynamics ${ }^{13}$ and ab initio Molecular Dynamics. ${ }^{14-16}$ At the single-molecule level, several electronic structure methods for the simulation of molecules under pressure are available. ${ }^{17}$ A particularly notable example is the eXtreme Pressure Polarizable Continuum Model (XP-PCM),${ }^{18-20}$ in which pressure is applied to a molecule via a surrounding medium. In XP-PCM, pressure is modeled by decreasing the size of the cavity inside which the molecule is placed and simultaneously increasing the Pauli repulsion of the surrounding medium. XP-PCM has been used, e.g., in the simulation of chemical reactions ${ }^{19-21}$ and for investigating changes in spectroscopic, ${ }^{22,23}$ electronic ${ }^{24}$ and structural ${ }^{25}$ properties of molecules under pressure.

A straightforward alternative to model molecules under pressure has its roots in quantum mechanochemistry. ${ }^{26}$ The earliest quantum mechanochemical method for the application of pressure is the Generalized Force-Modified Potential Energy Surface (G-FMPES) model, ${ }^{27-29}$ which builds on the FMPES scheme, ${ }^{30}$ a protocol to optimize molecular geometries under external forces. In G-FMPES, mechanical 
forces compress a molecule towards its geometric centroid during a geometry optimization. A variation of this method, the Hydrostatic Compression Force Field (HCFF) approach, ${ }^{31}$ differs from G-FMPES mainly in the definition of the surface area and the calculation of the pressure that is applied to the molecule. HCFF has been used to calculate the pressure required to trigger spin-crossover in octahedral metal ligand complexes. ${ }^{31}$ A drawback of the quantum mechanochemical pressure models, however, is that the pressure that is applied to the molecule cannot be defined a priori. Instead, the applied pressure is calculated from empirical parameters that are specified by the user, meaning that the desired pressure has to be adjusted iteratively. A second drawback is that in some cases, e.g. in extended molecules, unreasonable molecular geometries are found by G-FMPES and HCFF due to the tendency of the molecule to minimize its surface area under compression. Hence, both G-FMPES and HCFF work best for molecules that can be approximated as spherical.

In this paper, a new member of the family of mechanochemical pressure models, the eXtended Hydrostatic Compression Force Field (X-HCFF), is presented. It builds on the HCFF approach, but avoids the deficiencies of the previous mechanochemical pressure models in that 1) the user has full control over the pressure that is applied to the molecule, and 2) the pressure is applied truly hydrostatically, i.e. the compression of extended molecules can be simulated realistically. It is shown that structural parameters calculated with the novel X-HCFF model are of the same quality of those calculated with previous computational methods in both single molecules and molecular crystals and that experimental spectroscopic parameters can be reproduced accurately in the investigated test systems. Moreover, a pressure-induced chemical reaction is reproduced. The implementation of the X-HCFF approach is straightforward and only requires a surface tessellation routine, which is typically implemented in connection with implicit solvent models in many quantum chemical program packages, as well as a nuclear gradient code for the desired electronic structure method. While the calculations presented in this paper were all carried out with Density Functional Theory (DFT), ${ }^{32,33}$ wave-function based methods can of course be used instead. 
The computational cost of an X-HCFF calculation is virtually identical to the cost of a regular, pressurefree geometry optimization. The X-HCFF model will be available in a future release of the Q-Chem program package. ${ }^{34}$

The rest of this paper is structured as follows: After establishing the theoretical foundation of the $\mathrm{X}-\mathrm{HCFF}$ approach in Section 2, the performance of the method is tested in Section 3. In particular, comparisons are made against structural parameters of molecules under pressure calculated with $\mathrm{HCFF}$ (Section 3.1) and XP-PCM (Section 3.2). The performance of X-HCFF for the description of molecular crystals under pressure is tested by comparing against results calculated with periodic DFT (Section 3.3) as well as against experimental Raman spectra under pressure (Section 3.4). Finally, the dimerization of carbon dioxide under pressure is investigated in Section 3.5 to demonstrate the capability of X-HCFF to describe a simple pressure-induced chemical reaction. Possible routes for future developments are pointed out in Section 4.

\section{Theory}

\subsection{Theoretical background of HCFF}

As X-HCFF is based on the HCFF approach, the theoretical background of the latter shall be summarized first. In HCFF mechanical forces pointing towards the non-mass-weighted molecular centroid are applied on the nuclei to compress a molecule. ${ }^{31}$ As motivated in the literature for the closely related G-FMPES approach, ${ }^{27-29}$ the force acting on each atom is potentially unique, with the largest force acting on the outermost atom and the forces on the inner atoms being scaled by the distances of these atoms to the geometric centroid of the system. Using this condition as well as the definition of the pressure, $P=$ $F_{\perp} / A$, where $F_{\perp}$ is the normal component of the force at the surface and $A$ is the surface area, the 
magnitude of the force $f_{i}^{\mathrm{HCFF}}$ pushing an atom $i$ towards the molecular centroid is calculated in the HCFF scheme as

$$
\left|\boldsymbol{f}_{i}^{\mathrm{HCFF}}\right|=-P_{\text {guess }} \cdot A_{\mathrm{vdW}} \cdot \frac{r_{i}}{r_{\max }}
$$

where $P_{\text {guess }}$ is a user-defined guess for the pressure that is applied to the molecule, $A_{\mathrm{vdW}}$ is the molecular van-der-Waals surface area, $r_{i}$ is the distance of atom $i$ to the molecular centroid and $r_{\max }$ is the distance of the outermost atom to the centroid. The van-der-Waals surface is used as the definition of the molecular surface, since the surrounding medium, which applies pressure to the molecule, e.g. in a diamond-anvil cell, is in contact with the molecule at its van-der-Waals surface. The forces $f_{i}^{\mathrm{HCFF}}$ are then added to the nuclear gradient during a geometry optimization, leading to a compression of the molecule. As in other quantum mechanochemical optimization techniques, ${ }^{35}$ the optimization converges if the externally applied force (in this case all forces $\boldsymbol{f}_{i}^{\mathrm{HCFF}}$ ) and the internal restoring forces cancel. The pressure acting on the molecule has to be calculated a posteriori via

$$
P_{\mathrm{HCFF}}=\frac{\langle\|f\|\rangle}{A_{\mathrm{vdW}}},
$$

where $\langle\|f\|\rangle$ is the average force acting on the atoms. Typically, $P_{\text {guess }}$ overestimates $P_{\mathrm{HCFF}}$. Hence, the pressure that is applied to the molecule cannot be specified directly and instead needs to be calculated from a user-defined parameter, which is one of the major drawbacks of the mechanochemical models of pressure that have been developed. Moreover, since the forces are acting towards the molecular centroid, molecules tend to minimize their surface areas. As a result, unreasonable compressed geometries can ensue $^{31}$ and extended molecules can fold into sphere-like objects, which is not the expected behavior in hydrostatic compression experiments. 


\subsection{The X-HCFF approach}

Both problems are circumvented in the X-HCFF approach. The basic idea that pressure is mediated to the molecule via its van-der-Waals surface, e.g. during hydrostatic compression in a diamond-anvil cell, is carried over from HCFF to X-HCFF. In many quantum chemical program packages the van-der-Waals surface is calculated by superimposing atom-centered spheres, removing the overlapping regions and tessellating the surface using a Lebedev grid (Figure 1A). ${ }^{36}$ Instead of compressing the molecules towards its centroid, in X-HCFF truly hydrostatic compression is achieved by applying the forces perpendicular to the van-der-Waals surface in each tessellation point (Figure 1B).

Each surface tessera $j$ has an area $A_{j}$ and the van-der-Waals surface is calculated as $A_{\mathrm{vdW}}=\sum_{j}^{N_{\text {Tess }}} A_{j}$, where $N_{\text {Tess }}$ is the number of tessellation points. In X-HCFF, the force $\mathbf{f}_{i, j}$ acting on atom $i$ that stems from the pressure on the surface tessera $j$ acts along the connecting line between atom $i$ and tessera $j$ and points away from the latter (blue arrows in Figure 1B). Using again the definition of pressure, this force can be calculated via

$$
\mathbf{f}_{i, j}=-P \cdot A_{j} \cdot \frac{\left(\mathbf{r}_{i}-\mathbf{r}_{j}\right)}{\left|\mathbf{r}_{i}-\mathbf{r}_{j}\right|}
$$

where $P$ is the pressure that is applied to the molecule, $\mathbf{r}_{i}$ is the position of atom $i$ and $\mathbf{r}_{j}$ is the position of tessera $j$. The net force acting on atom $i$ is then calculated by summing up the contributions from each surface tessera that lies on the van-der-Waals sphere around atom $i$ (blue spheres in Figure 1B).

$$
\mathbf{f}_{i}=\sum_{j}^{N_{\text {Tess }}, i} \mathbf{f}_{i, j}
$$

When calculating the gradient contribution for an atom, the tesserae that lie on the van-der-Waals spheres around the other atoms are ignored. Since the force contributions from tesserae on opposite sides of the 

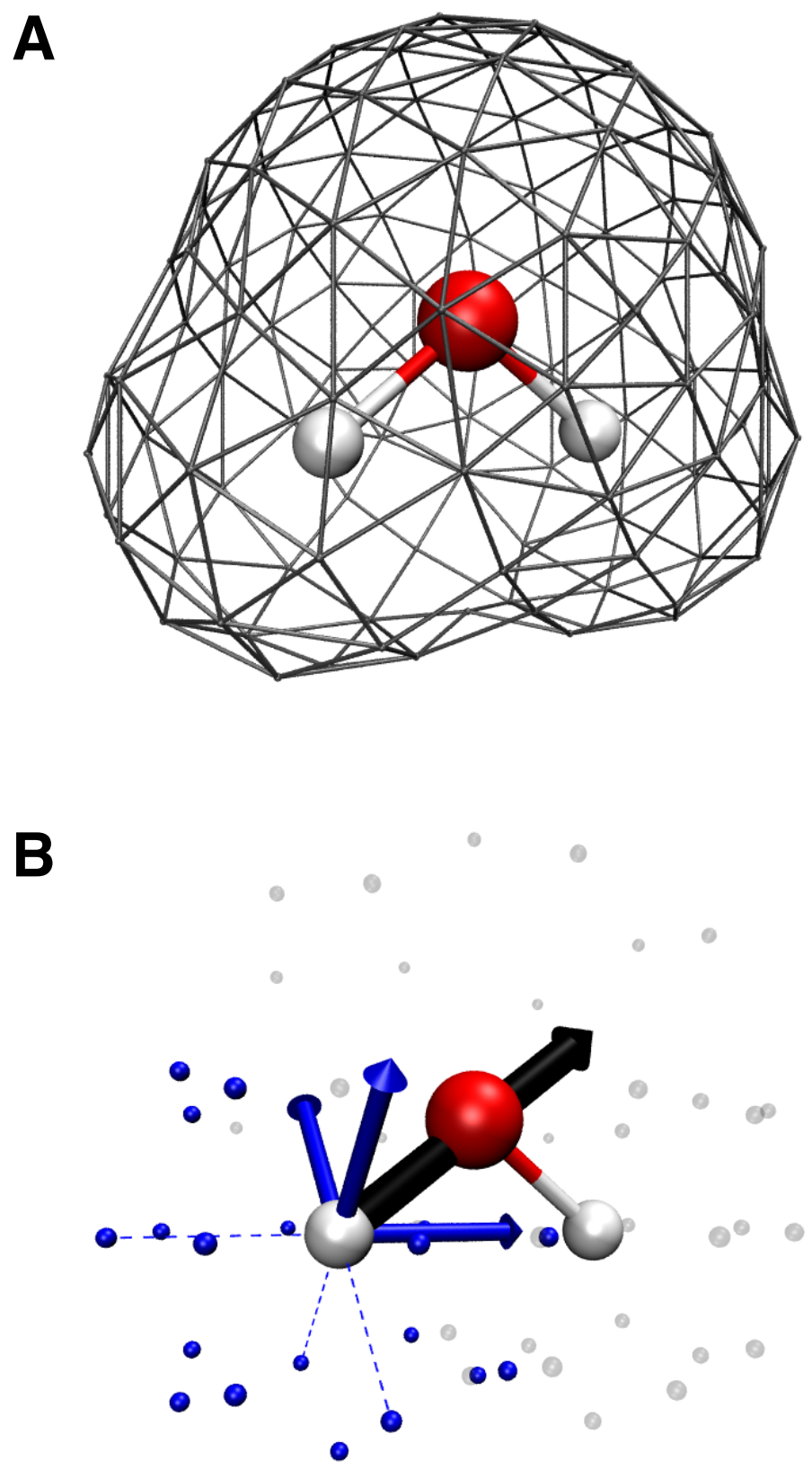

Figure 1: A) Van-der-Waals surface of the water molecule approximated by a Lebedev grid, leading to a tessellated surface. B) Basic idea of the X-HCFF approach for the water molecule with a reduced number of tessellation points, taking one of the hydrogen atoms as an example. The blue spheres represent the tessellation points created by the van-der-Waals sphere around the hydrogen atom under consideration, whereas the transparent spheres are the tessellation points associated with the other atoms in the molecule. Dotted lines connect the tessellation points to the hydrogen atom and the blue arrows depict the resulting gradient contributions. For clarity, the force contributions of only three tessellation points are shown. The thick black arrow represents the resulting force that arises when adding up each individual gradient contribution for the hydrogen atom. Arrows are not drawn to scale. 
atom cancel and there are no tessellation points along the connecting line between two atoms, the resulting force contribution pushes the atoms towards one another, leading to a compression of the molecule. The forces calculated for each atom via eq. 4 are added to the nuclear gradient during a geometry optimization, which converges when the external force due to pressure and the internal restoring force of the molecule cancel. Since the tessellation is carried out anew in each optimization step, the tessellation field reacts to the updated molecular geometry. As a result, the forces applied to the atoms are adjusted each time such that the input pressure $P$ is applied to the molecule. However, the $\mathrm{X}-\mathrm{HCFF}$ ansatz does not strictly guarantee that the gradient contributions on all atoms add up to zero in all cases, which is required to prevent translation and rotation of the molecule. While in all investigated systems the resulting net gradient contribution was extremely small, it was split up evenly and added to the nuclear gradient of each atom.

Since the van-der-Waals radii are not scaled in the X-HCFF approach, the only empirical parameter of the model is the number of tessellation points per atom. The dependence of the results on this parameter is tested in Section 3.2.

\section{Results and Discussion}

\subsection{Comparison between X-HCFF and HCFF}

To showcase the difference between X-HCFF and HCFF, an aliphatic molecule is compressed using both approaches. While HCFF was shown to deliver physically meaningful and chemically intuitive results for molecules that can be circumscribed onto a sphere, ${ }^{31}$ the method performs poorly for butane under a pressure of $50 \mathrm{GPa}$ (Figure 2): Since strong forces point towards the molecular centroid and these forces are strongest for the outermost atoms, the molecule tends to minimize its surface and folds into a 
HCFF:

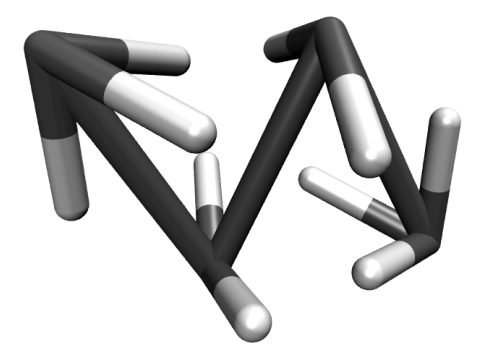

\section{X-HCFF:}

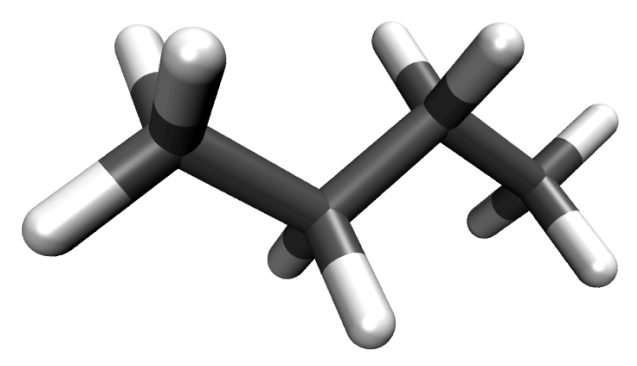

Figure 2: Molecular geometries for butane at a pressure of $50 \mathrm{GPa}$ as calculated with the $\mathrm{HCFF}^{31}$ (top) and the X-HCFF (bottom) approaches at the $\mathrm{PBE}^{37} / \mathrm{cc}-\mathrm{pVDZ}^{38}$ level of theory with 302 tessellation points for each atom.

sphere-like object. This situation is reminiscent of planets and stars, in which gravitational forces pull each particle towards the center, resulting in (roughly) spherical shapes. The creation of a sphere-like geometry is a general feature of HCFF and can also be observed in many other molecules, which limits the applicability of the method.

The X-HCFF approach, on the other hand, is not afflicted with this disadvantageous feature. While the bond lengths in the molecules are shortened by pressure and bond angles change (cf. the Supporting Information, Tables S1 and S2), the extended shape of butane is retained at $50 \mathrm{GPa}$ (Figure 2). This is due to the fact that each atom is being pushed inwards orthogonally to the surface, leading to a compression of the covalent bonds and not to the creation of a sphere-like geometry. Therefore, X-HCFF is more generally applicable for the simulation of molecules under hydrostatic pressure than HCFF. 


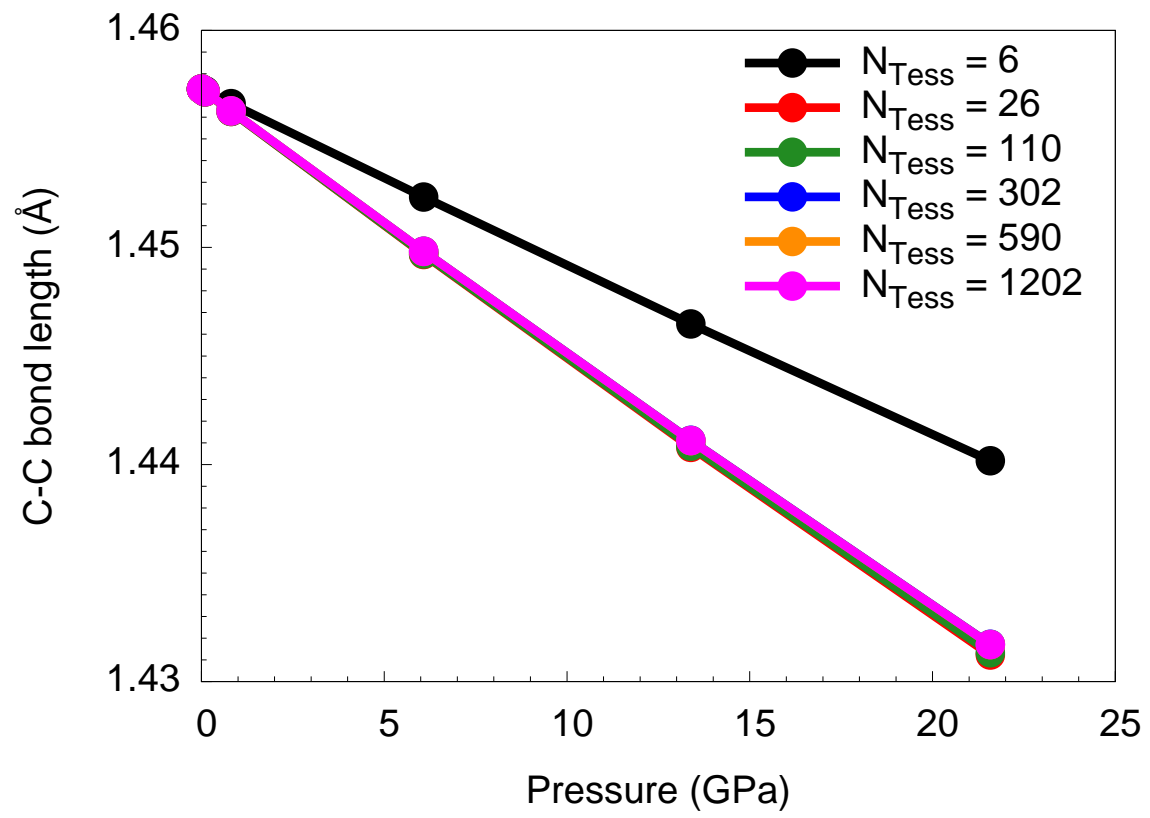

Figure 3: Dependence of the central $\mathrm{C}-\mathrm{C}$ bond length (single bond) in trans-1,3-butadiene on the number of tessellation points $\left(\mathrm{N}_{\text {Tess }}\right)$ per atom, calculated with X-HCFF at the B3LYP ${ }^{39-41} / 6-31 \mathrm{G}(\mathrm{d}, \mathrm{p})^{42}$ level of theory.

\subsection{Structural parameters in trans-1,3-butadiene as a function of pressure}

To judge the dependence of structural data calculated with X-HCFF on the number of tessellation points, trans-1,3-butadiene is used as a test case. Here, values between 6 and 1202 tessellation points per atom are adjusted. Focusing on the $\mathrm{C}-\mathrm{C}$ single bond length in trans-1,3-butadiene, it can be observed that, with the exception of the smallest number of tessellation points, the results converge quickly (Figure 3). With increasing pressure the $\mathrm{C}-\mathrm{C}$ bond length decreases with the same slope for all values between 26 and 1202 tessellation points per atom. Analogous results were obtained for all other investigated systems during testing. This is a profitable feature of X-HCFF, because the number of tessellation points is the only empirical parameter in the model and the results are virtually independent of the precise choice of this parameter. As the cost of the calculation is influenced only insignificantly by the number of tessellation points per atom, a value of 302 is used in the remainder of this paper.

Since trans-1,3-butadiene was treated previously with XP-PCM, ${ }^{18}$ this molecule provides a testing 
ground for the performance of X-HCFF in comparison to another computational method for the simulation of molecules under pressure. Comparing the bond lengths in trans-1,3-butadiene calculated with $\mathrm{XP}-\mathrm{PCM}$ and X-HCFF (Figure 4), it becomes apparent that the pressure-induced decrease in carboncarbon bond lengths is more pronounced in X-HCFF than in XP-PCM. Also, the X-HCFF curve for the central $\mathrm{C}-\mathrm{C}$ single bond is smoother than in XP-PCM. Turning to the carbon-hydrogen bond lengths, the results of X-HCFF and XP-PCM are more similar, with the difference that the bond lengths calculated with X-HCFF again decrease more smoothly. In particular, X-HCFF finds a monotonous decrease of one of the terminal $\mathrm{C}-\mathrm{H}$ bond lengths ( $\mathrm{C} 1-\mathrm{H} 5$ in Figure 4), while this is not the case for XP-PCM.

These results demonstrate that, using a single trans-1,3-butadiene molecule as an example, $\mathrm{X}-\mathrm{HCFF}$ affords continuous curves showing the progressive decrease of bond lengths with increasing pressure, in agreement with chemical intuition, and that the decrease in bond lengths is proportional to the external pressure. Extensive test calculations on a wide variety of molecules need to be carried out to judge how general this trend is.

\subsection{Structural parameters in a molecular crystal as a function of pressure}

The performance of X-HCFF when treating molecular crystals is tested by calculating structural parameters of 7-nitrotetrazolo [1,5]furazano[4,5-b]pyridine 1-oxide (NFP), for which reference values calculated with a periodic implementation of DFT are available. ${ }^{43}$ The focus in the present study lies on intramolecular geometric changes and not on changes in the lattice parameters, so that a single NFP molecule is subjected to pressures between 0 and $100 \mathrm{GPa}$ with the X-HCFF model. As can be seen in Figure 5,

the X-HCFF results can compete with those obtained via periodic DFT. Despite the differences in the approaches and the applied electronic structure levels, which lead to deviations in the bond lengths at $0 \mathrm{GPa}$, many of the bond lengths in NFP decrease with roughly the same slope in both approaches. No- 

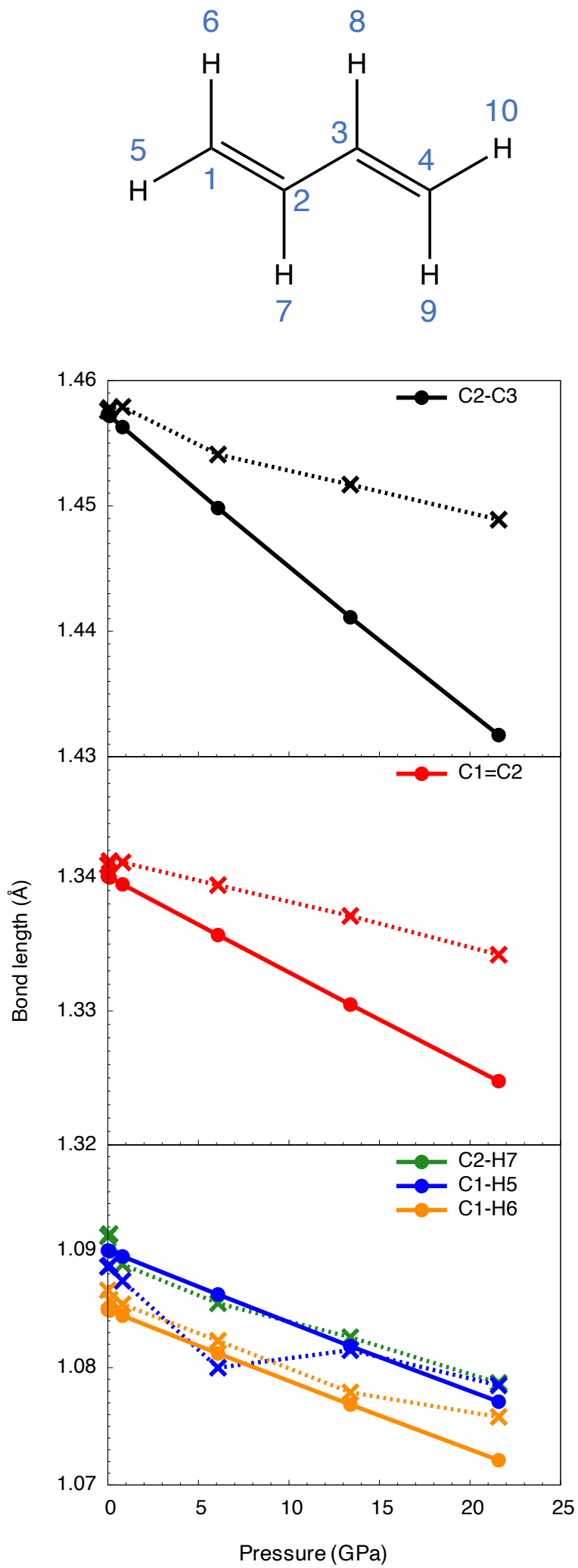

Figure 4: Bond lengths in trans-1,3-butadiene at different pressures. Circles connected by solid lines denote the X-HCFF results. Crosses connected by dotted lines represent literature values (ref. 18), calculated with XP-PCM. In both cases, B3LYP/6-31G(d,p) was taken as the electronic structure method. 


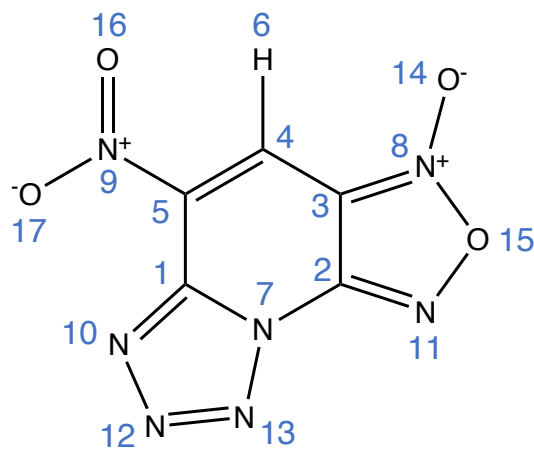

NFP

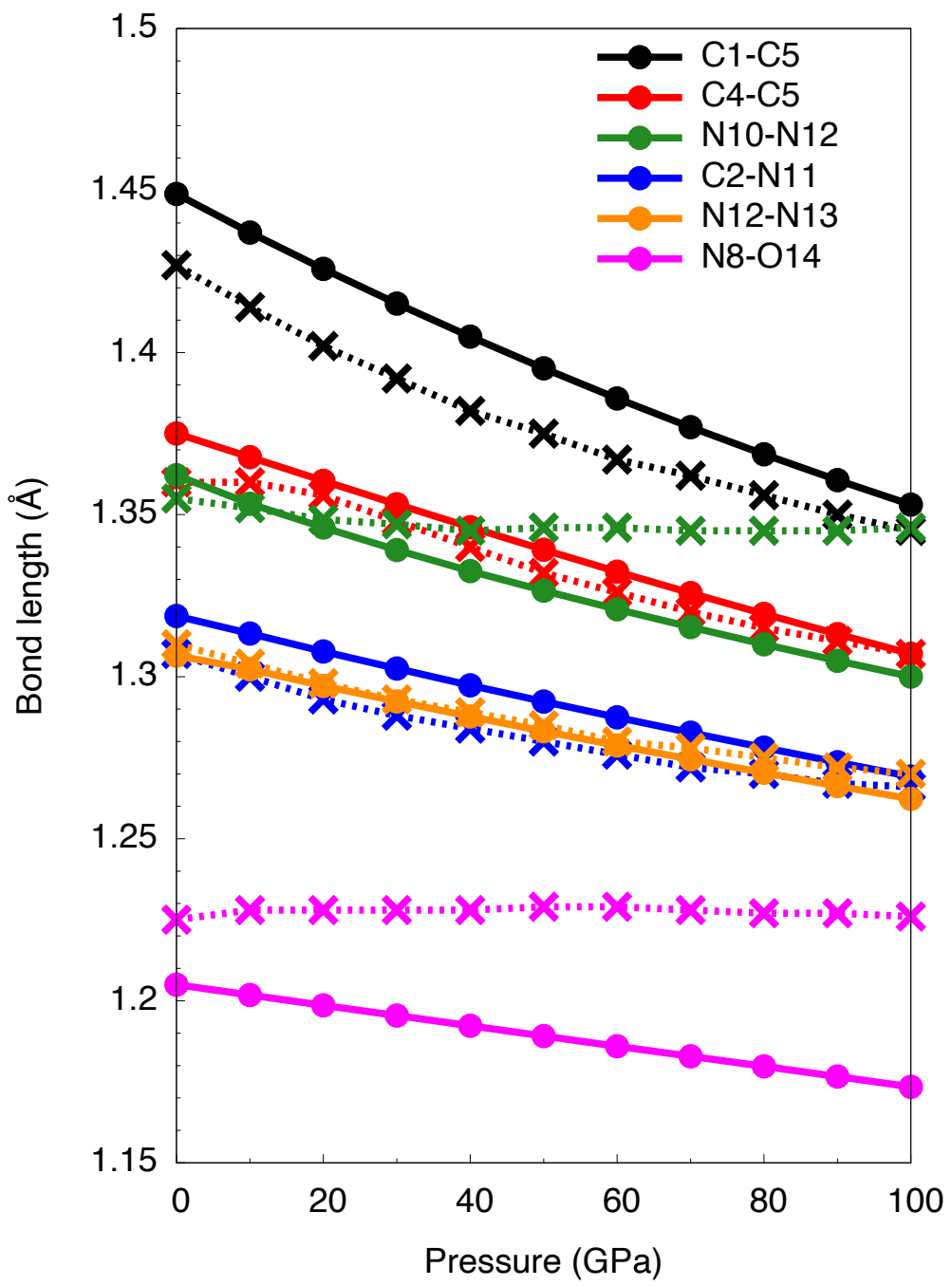

Figure 5: Selected bond lengths in NFP at different pressures. Circles connected by solid lines denote the X-HCFF values. Crosses connected by dotted lines represent literature values (estimated graphically from ref. 43), calculated with Density Functional Theory (DFT) for periodic systems. 
table differences include the bond lengths N10-N12 and N8-O14, which remain almost constant and even increase occasionally in the periodic calculations, whereas they decrease monotonously in X-HCFF. It can be hypothesized that these differences can be traced back to crystal packing effects that are not captured by X-HCFF. However, one can state that the structural parameters calculated with X-HCFF for the molecular NFP crystal are chemically intuitive and for many bond lengths the agreement with periodic calculations is remarkable, which is an encouraging result considering that only a single molecule is treated with X-HCFF. Nevertheless, in the future a careful calibration of the size of the system investigated with $\mathrm{X}-\mathrm{HCFF}$, i.e. the number of molecules subjected to pressure in the geometry optimization, needs to be carried out to determine the influence of the chemical environment in the crystal on the structural parameters of an individual molecule.

\subsection{Comparison with experimental Raman spectra under pressure}

Although comparisons of structural data of single molecules and molecular crystals calculated with XHCFF and other approaches are helpful to judge the performance of the new method, the clearest criterion for its reliability is the direct comparison with experimental data. Experimental structural data of molecules and crystals under pressure are typically inferred from spectroscopic observables. A notable example is the pressure-induced increase in the wavenumber of the Raman-active $\mathrm{C}-\mathrm{H}$ stretching vi-

bration in the molecular crystal 2-amine-1,3,4-thiadiazole (ATD), which has been reported recently. ${ }^{44}$ It was found that the wavenumber of this vibration in the ATD crystal increases linearly with increasing pressure.

Applying the same pressures as in the experiments to ATD with the X-HCFF approach at the PBE/ccpVDZ level of theory and subsequently calculating the Raman spectra yields virtually identical changes in the Raman spectrum (Figure 6), provided that the calculated wavenumbers are scaled with the ratio 


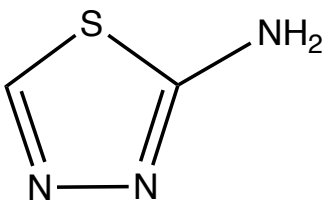

ATD

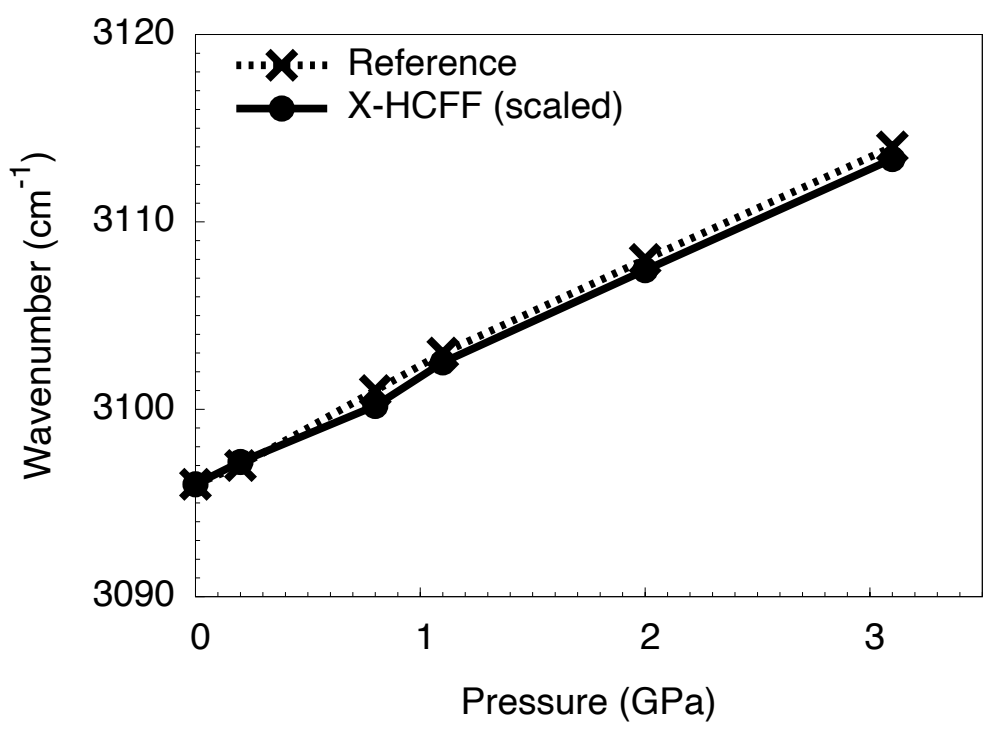

Figure 6: Wavenumbers of the Raman-active $\mathrm{C}-\mathrm{H}$ stretching vibration in ATD at different pressures, calculated with X-HCFF at the PBE/cc-pVDZ level of theory. The maxima of the peaks in the experimental Raman spectra presented in ref. 44 were taken as the reference values. The X-HCFF values were scaled such that the experimental and calculated peaks at $0 \mathrm{GPa}$ coincide.

between the experimental $\left(3096 \mathrm{~cm}^{-1}\right)$ and the calculated $\left(3171 \mathrm{~cm}^{-1}\right)$ signal at $0 \mathrm{GPa}$. The full set of scaled and unscaled calculated wavenumbers of the Raman-active $\mathrm{C}-\mathrm{H}$ stretching vibration in ATD at different pressures can be found in the Supporting Information (Table S4). Since experiments are free from the approximations made in other approaches for the simulation of molecules under pressure to which X-HCFF was compared in the previous Sections, the accurate reproduction of the experimental spectroscopic data presented here emphasizes the usefulness of X-HCFF in the simulation of chemical systems under pressure. The results suggest that both the experimental geometric changes and the accompanying response of the Raman spectra of the ATD crystal are captured accurately by X-HCFF. 


\subsection{Pressure-induced dimerization of carbon dioxide}

To test the applicability of the X-HCFF approach when describing pressure-induced chemical reactions, the dimerization of carbon dioxide under pressure is investigated as an example. This reaction has been described previously using ab initio Molecular Dynamics (AIMD) simulations ${ }^{45}$ and has been proposed as an intermediate step in the formation of polymeric $\mathrm{CO}_{2}$ under pressure.

Starting with a van-der-Waals complex of two $\mathrm{CO}_{2}$ molecules with a lateral distance of $3.15 \AA$ between each other, the application of pressure with X-HCFF at the PBE/cc-pVDZ level of theory leads to a continuous decrease in the distance between the two $\mathrm{CO}_{2}$ molecules up to $90 \mathrm{GPa}$ (Figure 7). Between 90 and $100 \mathrm{GPa}$, the distance between the $\mathrm{CO}_{2}$ molecules decreases sharply, marking the formation of covalent bonds in the resulting $\mathrm{CO}_{2}$ dimer. A further increase in pressure leads to only a minor compression of the $\mathrm{C}-\mathrm{O}$ bonds within the four-membered ring of the $\mathrm{CO}_{2}$ dimer. Interestingly, carrying out a pressure-free geometry optimization using the $\mathrm{CO}_{2}$ dimer created at $100 \mathrm{GPa}$ as an input structure,

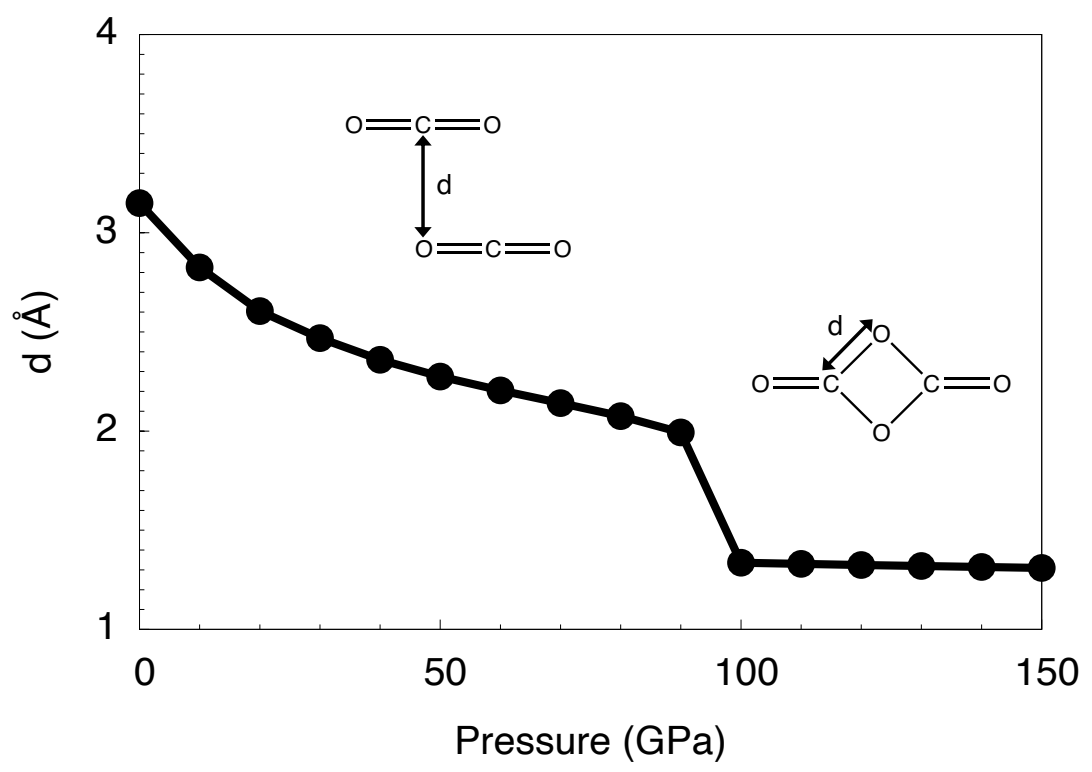

Figure 7: Distance $d$ between the carbon atom of a $\mathrm{CO}_{2}$ molecule and an oxygen atom of another $\mathrm{CO}_{2}$ molecule as a function of pressure, using X-HCFF at the PBE/cc-pVDZ level of theory. After dimerization occurs at approx. $100 \mathrm{GPa}, d$ denotes the $\mathrm{C}-\mathrm{O}$ bond length in the four-membered ring of the $\mathrm{CO}_{2}$ dimer. 
the dimeric geometry is retained, emphasizing the metastable nature of the dimer that was described previously. ${ }^{45}$ However, in the AIMD simulations the $\mathrm{CO}_{2}$ dimer was observed as a short-lived species at pressures of 20 and $50 \mathrm{GPa}$, which is lower than in the X-HCFF calculations. A possible reason for the higher pressure required to form the $\mathrm{CO}_{2}$ dimer using X-HCFF is that, in contrast to AIMD simulations, thermal oscillations are not taken into account. Such fluctuations, however, lead to molecular oscillations and could deliver crucial energy required to overcome the reaction barrier. Similar effects have been discussed as the reason for the overestimation of rupture forces by static quantum chemical

methods when describing mechanochemical processes. ${ }^{26}$ Detailed calculations will need to be carried out to reliably compare the pressures required to induce chemical reactions calculated by dynamic and static simulations techniques.

The pressure-induced dimerization of $\mathrm{CO}_{2}$, which was successfully described with X-HCFF, demonstrates that the predictive power of the new method is not limited to pressure-dependent structural and spectroscopic properties, but also has high potential in describing chemical reactions under pressure. In this case, a minimum on the potential energy surface under pressure, which was revealed by previous AIMD simulations, could be reproduced at the computational cost of a quantum chemical geometry optimization.

\section{Conclusions and Outlook}

In this paper, the eXtended Hydrostatic Compression Force Field (X-HCFF), a novel mechanochemical method for the simulation of molecules and molecular crystals under hydrostatic pressure, was introduced. The X-HCFF method avoids common problems of related approaches and accurately reproduces computational and experimental structural and spectroscopic reference data in a variety of test systems. The straightforward implementation and the user-friendly and inexpensive computation make X-HCFF a 
useful method for calculations of structural and spectroscopic properties as well as chemical transformations of molecules and molecular crystals under hydrostatic pressure.

In the future, careful benchmarks of various density functionals and wave-function based methods will be carried out to determine the optimal electronic structure method that reproduces experimental structural and spectroscopic data most accurately. Moreover, it is planned to leave the realm of small test systems and evaluate the applicability of X-HCFF when treating crystallites. A particularly interesting question is whether pressure-induced changes in lattice constants ${ }^{9-11}$ can be reproduced by using a quantum mechanochemical method. The ability of X-HCFF to go beyond the treatment of single molecules and deliver chemically meaningful results for the dimerization of carbon dioxide is very promising in this regard. Another future route is provided by AIMD simulations, with which X-HCFF can be combined easily, to simulate the time evolution of structural changes of molecules under pressure. This will be particularly useful in modeling pressure-induced adsorption and desorption processes of, e.g., industrially relevant gases on surfaces. Furthermore, more complex chemical reactions under pressure, e.g. Diels-Alder reactions, will be treated with X-HCFF in the future.

Finally, it is important to note that in the X-HCFF approach molecules react towards hydrostatic pressure only through force-induced compression of the nuclear scaffold. While this opens up the interesting possibility of relating the strain induced by the compression with chemical reactivity, ${ }^{46}$ one must acknowledge that pressure does not interact directly with the electrons, but only indirectly through an altered arrangement of the nuclei. A computational method that simulates the influence of pressure on molecules via interactions with the electron density is currently under development. 


\section{Acknowledgements}

The author would like to thank M. Scheurer, University of Heidelberg, for helpful discussions and for proofreading of the manuscript. The idea for this project was conceived during the work on a different electronic structure method for the simulation of molecules under hydrostatic pressure, which is currently being developed by M. Scheurer, A. Dreuw (University of Heidelberg), E. Epifanovsky (Q-Chem Inc.), M. Head-Gordon (University of California, Berkeley) and the author of this paper.

\section{References}

[1] McMillan, P. F. Chemistry at high pressure. Chem. Soc. Rev. 2006, 35, 855-857.

[2] Grochala, W.; Hoffmann, R.; Feng, J.; Ashcroft, N. W. The Chemical Imagination at Work in Very Tight Places. Angew. Chem. Int. Ed. 2007, 46, 3620-3642.

[3] Schettino, V.; Bini, R. Constraining molecules at the closest approach: chemistry at high pressure. Chem. Soc. Rev. 2007, $36,869-880$.

[4] Li, B.; Ji, C.; Yang, W.; Wang, J.; Yang, K.; Xu, R.; Liu, W.; Cai, Z.; Chen, J.; kwang Mao, H. Diamond anvil cell behavior up to 4 Mbar. Proc. Nat. Acad. Sci. U.S.A. 2018, 115, 1713-1717.

[5] Stixrude, L.; Cohen, R. E. High-Pressure Elasticity of Iron and Anisotropy of Earth's Inner Core. Science 1995, 267, 1972-1975.

[6] Casati, N.; Kleppe, A.; Jephcoat, A. P.; Macchi, P. Putting pressure on aromaticity along with in situ experimental electron density of a molecular crystal. Nat. Commun. 2016, 7, 10901.

[7] Chandrasekhar, M.; Guha, S.; Graupner, W. Squeezing Organic Conjugated Molecules - What Does One Learn? Adv. Mater. 2001, 13, 613-618.

[8] Kato, M.; Higashi, M.; Taniguchi, Y. Effect of pressure on the internal rotation angle of biphenyl in carbon disulfide. $J$. Chem. Phys. 1988, 89, 5417-5421.

[9] Puschnig, P.; Ambrosch-Draxl, C.; Heimel, G.; Zojer, E.; Resel, R.; Leising, G.; Kriechbaum, M.; Graupner, W. Pressure studies on the intermolecular interactions in biphenyl. Synth. Met. 2001, 116, 327-331.

[10] Katrusiak, A. High-Pressure X-Ray Diffraction Study Of 2-Methyl-1,3-Cyclopentanedione Crystals. High Press. Res. 1991, 6, 155-167.

[11] Cartz, L.; Srinivasa, S. R.; Riedner, R. J.; Jorgensen, J. D.; Worlton, T. G. Effect of pressure on bonding in black phosphorus. J. Chem. Phys. 1979, 71, 1718-1721.

[12] Demontis, P.; Lesar, R.; Klein, M. L. New High-Pressure Phases of Ice. Phys. Rev. Lett. 1988, 60, 2284-2287.

[13] Nosé, S.; Klein, M. L. Constant pressure molecular dynamics for molecular systems. Mol. Phys. 1983, 50, 1055-1076.

[14] Mugnai, M.; Cardini, G.; Schettino, V. Charge separation and polymerization of hydrocarbons at an ultrahigh pressure. Phys. Rev. B 2004, 70, 020101. 
[15] Imoto, S.; Kibies, P.; Rosin, C.; Winter, R.; Kast, S. M.; Marx, D. Toward Extreme Biophysics: Deciphering the Infrared Response of Biomolecular Solutions at High Pressures. Angew. Chem. Int. Ed. 2016, 55, 9534-9538.

[16] Marx, D.; Hutter, J. Ab Initio Molecular Dynamics. Basic Theory and Advanced Methods, 1st ed.; Cambridge University Press: Cambridge, UK, 2009.

[17] Stauch, T. Quantum chemical modeling of molecules under pressure. Int. J. Quantum Chem. 2020, e26208.

[18] Cammi, R.; Verdolino, V.; Mennucci, B.; Tomasi, J. Towards the elaboration of a QM method to describe molecular solutes under the effect of a very high pressure. Chem. Phys. 2008, 344, 135-141.

[19] Cammi, R. A New Extension of the Polarizable Continuum Model: Toward a Quantum Chemical Description of Chemical Reactions at Extreme High Pressure. J. Comput. Chem. 2015, 36, 2246-2259.

[20] Chen, B.; Hoffmann, R.; Cammi, R. The Effect of Pressure on Organic Reactions in Fluids - a New Theoretical Perspective. Angew. Chem. Int. Ed. 2017, 56, 11126-11142.

[21] Fukuda, R.; Nakatani, K. Quantum Chemical Study on the High-Pressure Effect for [4 + 4] Retrocycloaddition of Anthracene Cyclophane Photodimer. J. Phys. Chem. C 2019, 123, 4493-4501.

[22] Pagliai, M.; Cardini, G.; Cammi, R. Vibrational Frequencies of Fullerenes C60 and C70 under Pressure Studied with a Quantum Chemical Model Including Spatial Confinement Effect. J. Phys. Chem. A 2014, 118, 5098-5111.

[23] Fukuda, R.; Ehara, M.; Cammi, R. Modeling molecular systems at extreme pressure by an extension of the polarizable continuum model (PCM) based on the symmetry-adapted cluster-configuration interaction (SAC-CI) method: Confined electronic excited states of furan as a test case. J. Chem. Theory Comput. 2015, 11, 2063-2076.

[24] Rahm, M.; Cammi, R.; Ashcroft, N. W.; Hoffmann, R. Squeezing All Elements in the Periodic Table: Electron Configuration and Electronegativity of the Atoms under Compression. J. Am. Chem. Soc. 2019, 141, 10254-10271.

[25] Caratelli, C.; Cammi, R.; Chelli, R.; Pagliai, M.; Cardini, G.; Schettino, V. Insights on the Realgar Crystal under Pressure from XP-PCM and Periodic Model Calculations. J. Phys. Chem. A 2017, 121, 8825-8834.

[26] Stauch, T.; Dreuw, A. Advances in Quantum Mechanochemistry: Electronic Structure Methods and Force Analysis. Chem. Rev. 2016, 116, 14137-14180.

[27] Subramanian, G.; Mathew, N.; Leiding, J. A generalized force-modified potential energy surface for mechanochemical simulations. J. Chem. Phys. 2015, 143, 134109.

[28] Jha, S. K.; Brown, K.; Todde, G.; Subramanian, G. A mechanochemical study of the effects of compression on a DielsAlder reaction. J. Chem. Phys. 2016, 145, 074307.

[29] Todde, G.; Jha, S. K.; Subramanian, G. The effect of external forces on the initial dissociation of RDX (1,3,5-trinitro1,3,5-triazine): A mechanochemical study. Int. J. Quantum Chem. 2017, 117, e25426.

[30] Ong, M. T.; Leiding, J.; Tao, H.; Virshup, A. M.; Martínez, T. J. First Principles Dynamics and Minimum Energy Pathways for Mechanochemical Ring Opening of Cyclobutene. J. Am. Chem. Soc. 2009, 131, 6377-6379.

[31] Stauch, T.; Chakraborty, R.; Head-Gordon, M. Quantum Chemical Modeling of Pressure-Induced Spin Crossover in Octahedral Metal-Ligand Complexes. ChemPhysChem 2019, 20, 2742-2747.

[32] Hohenberg, P.; Kohn, W. Inhomogeneous Electron Gas. Phys. Rev. 1964, 136, 864-871.

[33] Kohn, W.; Sham, L. J. Self-Consistent Equations Including Exchange and Correlation Effects. Phys. Rev. 1965, 140, $1133-1138$.

[34] Shao, Y. et al. Advances in molecular quantum chemistry contained in the Q-Chem 4 program package. Mol. Phys. 2014, 113, 184-215. 
[35] Kochhar, G. S.; Heverly-Coulson, G. S.; Mosey, N. J. Theoretical Approaches for Understanding the Interplay Between Stress and Chemical Reactivity. Top. Curr. Chem. 2015, 369, 37-96.

[36] Lange, A. W.; Herbert, J. M. Polarizable Continuum Reaction-Field Solvation Models Affording Smooth Potential Energy Surfaces. J. Phys. Chem. Lett. 2010, 1, 556-561.

[37] Perdew, J. P.; Burke, K.; Ernzerhof, M. Generalized Gradient Approximation Made Simple. Phys. Rev. Lett. 1996, 77, 3865-3868.

[38] Dunning, T. H. Gaussian basis sets for use in correlated molecular calculations. I. The atoms boron through neon and hydrogen. J. Chem. Phys. 1989, 90, 1007-1023.

[39] Becke, A. D. Density-functional exchange-energy approximation with correct asymptotic behavior. Phys. Rev. A 1988, $38,3098-3100$.

[40] Lee, C.; Yang, W.; Parr, R. G. Development of the Colle-Salvetti correlation-energy formula into a functional of the electron density. Phys. Rev. B 1988, 37, 785-789.

[41] Becke, A. D. A new mixing of Hartree-Fock and local density-functional theories. J. Chem. Phys. 1993, 98, 1372-1377.

[42] Hehre, W. J.; Ditchfield, R.; Pople, J. A. Self-Consistent Molecular Orbital Methods. XII. Further Extensions of Gaussian-Type Basis Sets for Use in Molecular Orbital Studies of Organic Molecules. J. Chem. Phys. 1972, 56, 2257-2261.

[43] Liu, H.; Wang, F.; Gong, X. DFT studies on 7-nitrotetrazolo [1,5]furazano[4,5-b]pyridine 1-oxide: Crystal structure, detonation properties, sensitivity and effect of hydrostatic compression. Struct. Chem. 2014, 25, 239-249.

[44] de Toledo, T. A.; da Costa, R. C.; Bento, R. R. F.; Pizani, P. S. Hydrostatic pressure and temperature effect on the Raman spectra of the molecular crystal 2-amine-1,3,4-thiadiazole. J. Mol. Struct. 2018, 1156, 127-135.

[45] Tassone, F.; Chiarotti, G. L.; Rousseau, R.; Scandolo, S.; Tosatti, E. Dimerization of CO2 at High Pressure and Temperature. ChemPhysChem 2005, 6, 1752-1756.

[46] Stauch, T.; Dreuw, A. Quantum Chemical Strain Analysis For Mechanochemical Processes. Acc. Chem. Res. 2017, 50, 1041-1048. 